\title{
Caveolin-1 expression in human breast lobular cancer progression
}

\author{
Giuseppe Perrone ${ }^{1,4}$, Vittorio Altomare ${ }^{2,4}$, Mariagiovanna Zagami $^{1}$, Sergio Morini ${ }^{3}$, \\ Tommaso Petitti ${ }^{3}$, Cleonice Battista ${ }^{2}$, Andrea Onetti Muda ${ }^{1}$ and Carla Rabitti ${ }^{1}$ \\ ${ }^{1}$ Department of Anatomical Pathology, Universitá Campus Bio-Medico di Roma, Rome, Italy; ${ }^{2}$ Breast Surgical \\ Unit, Universitá Campus Bio-Medico di Roma, Rome, Italy and ${ }^{3}$ Department of Biomedical Research, \\ Universitá Campus Bio-Medico di Roma, Rome, Italy
}

\begin{abstract}
Caveolin-1 is the principal structural protein of caveolae, and caveolin-1 gene plays a role as a tumour suppressor gene in human mammary cancer-derived cells. However, limited data are available concerning caveolin-1 expression in human breast cancer tissue. We evaluated caveolin-1 expression in normal lobular epithelial cells and in the whole human lobular neoplasia spectrum disease, with the aim to examine differences of caveolin-1 expression in human lobular neoplasia progression. We selected 147 cases of pure lobular lesions, ie lobular intraepithelial neoplasia and invasive lobular carcinoma, from 112 patients. Presence of caveolin-1 was evaluated by immunohistochemistry. Among 81 lobular intraepithelial neoplasia lesions studied, $43 \%$ were associated with invasive lobular carcinoma, with positive correlation between lobular intraepithelial neoplasia grade and presence of invasive component $(P=0.01)$. In total, $64 \%$ of lobular lesions were positive for caveolin-1 (81\% lobular intraepithelial neoplasia and $42 \%$ invasive lobular carcinoma), and a significant difference in terms of caveolin-1 expression was present between lobular intraepithelial neoplasia and invasive lobular carcinoma $(P=0.0001)$. Variations in caveolin-1 expression were evident among the different lobular intraepithelial neoplasia grades $(91 \%$ grade $1,68 \%$ grade $2,35 \%$ grade 3$)$; the difference was significant comparing lobular intraepithelial neoplasia grade 3 vs $1(P=0.0001)$ and grade 3 vs $2(P=0.007)$ but not grade 1 vs 2 . Furthermore, significant differences were found between lobular intraepithelial neoplasia grades 1 and 2 vs invasive lobular carcinoma $(P=0.0001)$, but not between lobular intraepithelial neoplasia grade 3 and invasive lobular carcinoma $(P=0.196)$. In conclusion, variations of caveolin-1 expression may have an important role in the progression of human breast lobular cancer; in addition, they confirm the powerful clinical impact of the lobular intraepithelial neoplasia classification for lobular intraepithelial neoplasia, supporting the direct origin of invasive lobular carcinoma from clonal expansion of the lobular intraepithelial neoplasia lesions cells.
\end{abstract}

Modern Pathology (2009) 22, 71-78; doi:10.1038/modpathol.2008.154; published online 3 October 2008

Keywords: breast; caveolin-1; immunohistochemistry, lobular intraepithelial neoplasia, invasive lobular cancer

Caveolins are the major integral membrane component of caveolae, flask-shaped invaginations of the plasma membrane with an average diameter of $50-100 \mathrm{~nm} .{ }^{1}$ In particular, caveolin-1 (Cav-1) is the principal structural protein of caveolae, ${ }^{1,2}$ and it is normally expressed at high levels in adipocytes, stromal cells and in normal mammary epithelia. ${ }^{3-6}$ Cav-1 appears to have diverse functions, including vesicular transport, maintaining cellular cholesterol

Correspondence: Dr G Perrone, MD, Department of Anatomical Pathology, Universitá Campus Bio-Medico di Roma, Via Alvaro del Portillo 200, Rome 00128, Italy.

E-mail: g.perrone@unicampus.it

${ }^{4}$ These authors contributed equally to the work.

Received 4 June 2008; revised and accepted 26 August 2008; published online 3 October 2008 balance, and signal transduction. ${ }^{7}$ Interestingly, Cav-1 gene is localized to the D7S522 locus in the q31.1 region of human chromosome $7 .^{6,8-10}$ This chromosomal region (D7S522/7q31.1) is a suspected tumour suppressor locus and a common fragile site (known as FRA7G) that is frequently deleted in a number of human cancers. ${ }^{11-16}$ Thus, Cav-1 may be the as yet unidentified tumour suppressor gene located at the D7S522/7q31.1 locus. ${ }^{8-10}$

In addition, Cav-1 has demonstrated an important role in mammary gland physiology and morphology. Recently, two novel phenotypes was described in the mammary glands of Cav-1 null mice: (1) mild mammary epithelial cell hyperplasia in virgin mice, ${ }^{17}$ and (2) precocious lactation in pregnant mice. ${ }^{18}$ Moreover, during pregnancy, Cav-1 null mice demonstrated accelerated development of the 
lobuloalveolar compartment, premature lactation, and hyperactivation of the Jak-2/STAT5a signalling cascade. ${ }^{19}$

Interestingly, data about the role of Cav-1 expression in the onset or progression of human breast cancers were also obtained. By using cultured human mammary epithelial cells, normal mammary epithelial cells expressed significant levels of Cav-1. ${ }^{3-6,8}$ Sager et $a l^{4}$ identified Cav-1 as one of 26 genes downregulated in human mammary adenocarcinoma-derived cells through differential display and subtractive hybridization techniques. Independently, Lee et $a l^{3}$ demonstrated that a number of human breast cancer cell lines show a significant reduction in Cav-1 expression levels, compared with normal mammary epithelial cells. Additionally, reintroduction of Cav-1 resulted in a $\sim 50 \%$ reduction in cellular proliferation and an $\sim 15$-fold reduction in the ability of these cells to form colonies in soft agar. ${ }^{3}$

To date, only limited studies have investigated the Cav-1 expression in human breast cancer tissue; Park et $a l^{20}$ by immunohistochemical methods, demonstrated a reduction in Cav-1 expression in invasive ductal carcinoma of the breast compared with normal mammary epithelia. Furthermore, our group recently demonstrated the expression of Cav-1 protein in a large portion of human lobular intraepithelial neoplasia of the breast. ${ }^{21}$ In the present study, the Cav-1 expression was evaluated in normal lobular epithelial cells and in the human lobular neoplasia spectrum (lobular intraepithelial neoplasia, invasive lobular cancer) with the aim to comprehensively examine differences of Cav-1 expression in human lobular neoplasia progression.

\section{Materials and methods}

\section{Clinical Material}

Immunohistochemical analysis was retrospectively performed on tissue samples that were taken for routine diagnostic purposes. On the basis of the availability of tissue samples in the archives of the Anatomical Pathology Department of the Campus Bio-Medico University of Rome, 112 patients with lobular neoplasia of the breast (lobular intraepithelial neoplasia lesions or invasive lobular carcinomas) between January 2001 and June 2007 were identified and analysed. No patient had received chemotherapy, hormonotherapy or radiation therapy before surgery. Tissue samples were fixed in $10 \%$ neutral-buffered formaldehyde and embedded in paraffin. Routine haematoxylin and eosin staining was performed on the sections for histopathologic evaluation. Lesions histology and lesions grade evaluated at primary diagnosis and extracted from pathology reports were re-evaluated by one of the authors (MZ). For lobular intraepithelial neoplasia, a three-tiered grading system proposed by Bratthauer and Tavassoli ${ }^{22}$ was used. Table 1 summarized the histological criteria. On the basis of the quality of the morphologic preservation of all available haematoxylin and eosin-stained slides of the surgical specimen sections, we selected one paraffin block for each case. Consecutive $3 \mu \mathrm{m}$ sections were re-cut from each study block. These sections were immunostained for Cav-1 proteins.

\section{Immunohistochemical Staining}

Immunohistochemistry was performed by the streptoavidin-biotin method. Endogenous peroxidase in the section was blocked by incubating them in $3 \%$ hydrogen peroxide. The used antibody was a rabbit polyclonal antibody against Cav-1 protein $(\mathrm{N}-20$, Santa Cruz Biotechnology Inc.) at a 1:100 dilution. This antibody has been used and validated previously by our group and by others. ${ }^{21,23,24}$ Sections were incubated with LSAB2 (Dakocytomation). 3-3'diaminobenzidine was used for colour development and haematoxylin was used for counterstaining. Negative control slides processed without primary antibody were included for each staining. Slides were examined by two investigators (GP and MZ) without knowledge of the corresponding clinicopathologic data.

Immunostaining was considered positive if appropriate brown staining was seen in membrane or cytoplasm of tumour cell. Cav-1 expression was established calculating the percentage of immunoreactive cells in a total of 1000 neoplastic cells. On the basis of percentage of Cav-1-positive cells, lesions were arbitrarily classified in: $0-25 \%$ (negative), 26-50\% (low), 51-75 (moderate), 76-100 (high). Agreement in immunohistochemical evaluation between the two observers was $>90 \%$. In cases of disagreement, a final score was determined by consensus after re-examination.

\section{Statistical Analysis}

Caveolin-1 expression among different grade of lobular intraepithelial neoplasia lesions and invasive lobular carcinoma was compared according to the Mann-Whitney $U$-tests for non-parametric independent variables. Spearman's rank correlation test was used to assess association between lobular intraepithelial neoplasia and invasive lobular carcinoma. $P<0.05$ was regarded as statistically significant in tailed tests. SPSS software (version 14.00; SPSS Inc., Chicago, IL, USA) was used for statistical analysis.

\section{Results}

\section{Patients' Characteristics}

One hundred-twelve patients with diagnosis of lobular neoplasia of the breast without other neoplastic lesions associated were studied. Our 
Table 1 Histological criteria proposed by Bratthauer and Tavassoli for grading lobular intraepithelial neoplasia

LIN, grade 1

Loosely cohesive cellular proliferation

Preserving distinct ductular outlines

Filling the acinar space

Acinar distension

Nuclear pleomorphism ${ }^{\mathrm{a}}$

Pure classic signet ring cell population ${ }^{\mathrm{a}}$
LIN, grade 2

$\begin{array}{ccc}+ & + & + \\ + & + & -(\text { Virtual confluence) } \\ \pm & + & + \\ - & + & +++ \text { (Maximally) } \\ - & - & \text { Rarely } \\ - & - & \text { Rarely }\end{array}$

LIN, lobular intraepithelial neoplasia.

${ }^{\mathrm{a}}$ If present, maximum distension of acini is not required.

Table 2 Association between lobular intraepithelial neoplasia and invasive lobular carcinoma

\begin{tabular}{lccc}
\hline & & LIN without ILC (\%) & LIN with ILC (\%) \\
\hline LIN grade 1 & 23 & $17(74)$ & $6(26)$ \\
LIN grade 2 & 38 & $22(58)$ & $16(42)$ \\
LIN grade 3 & 20 & $7(35)$ & $13(65)$ \\
Total & 81 & $46(57)$ & $35(43)$ \\
\hline
\end{tabular}

LIN, lobular intraepithelial neoplasia; ILC, invasive lobular carcinoma. Spearman's test: $r=0.284 ; P=0.01$.

cohort was formed by tree different population of lobular breast neoplasia: (1) 46 lobular intraepithelial neoplasia lesions without invasive lobular cancer component, (2) 35 lobular intraepithelial neoplasia lesions with invasive lobular carcinoma component and (3) 31 invasive lobular carcinomas without in situ component. Within the patients with invasive carcinoma, 25 had lymph node metastasis.

\section{Relationship Between Lobular Intraepithelial Neoplasia and Invasive Lobular Carcinoma}

Analysing the association between lobular intraepithelial neoplasia and invasive lobular carcinoma, we found that among 81 lobular intraepithelial neoplasia lesions studied, $35(43 \%)$ showed to be associated to invasive lobular carcinoma. Moreover, a positive significant correlation was found between lobular intraepithelial neoplasia grade and the presence of invasive component $(r=0.284 ; P=0.01)$, demonstrating that the increasing grade of lobular intraepithelial neoplasia directly correlates with association of invasive lobular carcinoma (Table 2, Figure 1).

\section{Immunohistochemical Staining for Cav-1}

Caveolin-1 immunostaining showed a prevalent membrane pattern associated to a cytoplasmic positivity in the normal ductal and lobular epithelial cells and surrounding endothelial cells in all 112 tissue examined. In the cases with concurrent lobular intraepithelial neoplasia lesion and invasive lobular carcinoma, the evaluation of Cav-1 was performed separately in the two different lesions.

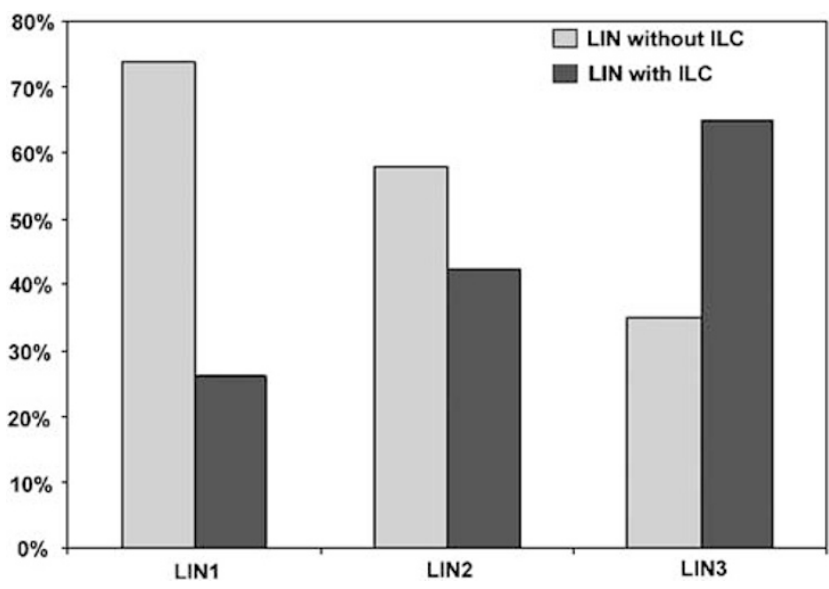

Figure 1 Relationship between lobular intraepithelial neoplasia and invasive lobular carcinoma. The increasing grade of lobular intraepithelial neoplasia directly correlates with association of invasive lobular carcinoma $(P=0.01)$.

Thus, we analysed 147 lobular lesions. The evaluation of Cav-1 expression in the lobular neoplasia samples showed that 94 of 147 lesions were positive for Cav-1. In particular, 66 of 81 lobular intraepithelial neoplasia (40 of 46 without and 26 of 35 with invasive component) and 28 of 66 invasive lobular carcinoma (10 of 31 without and 18 of 35 with in situ component) showed Cav-1 expression (Figure 2).

\section{Cav-1 in Lobular Intraepithelial Neoplasia Lesions}

No significant differences $(P=0.835)$ in terms of Cav-1 expression emerged between lobular intraepithelial neoplasia lesions with and without invasive component. Moreover, stratifying lobular intraepithelial neoplasia in a three-tiered grading system, no significant differences resulted between lobular intraepithelial neoplasia with and without invasive component (Table 3 ).

Thus, the complete absence of statistically significant differences between lobular intraepithelial neoplasia with and without invasive lobular carcinoma, suggests that these two populations are not different in terms of Cav-1 expression. On the bases of these findings, to compare Cav-1 expression in the different grades of lobular intraepithelial neoplasia, the 81 lobular intraepithelial neoplasia lesions were examined all together: 

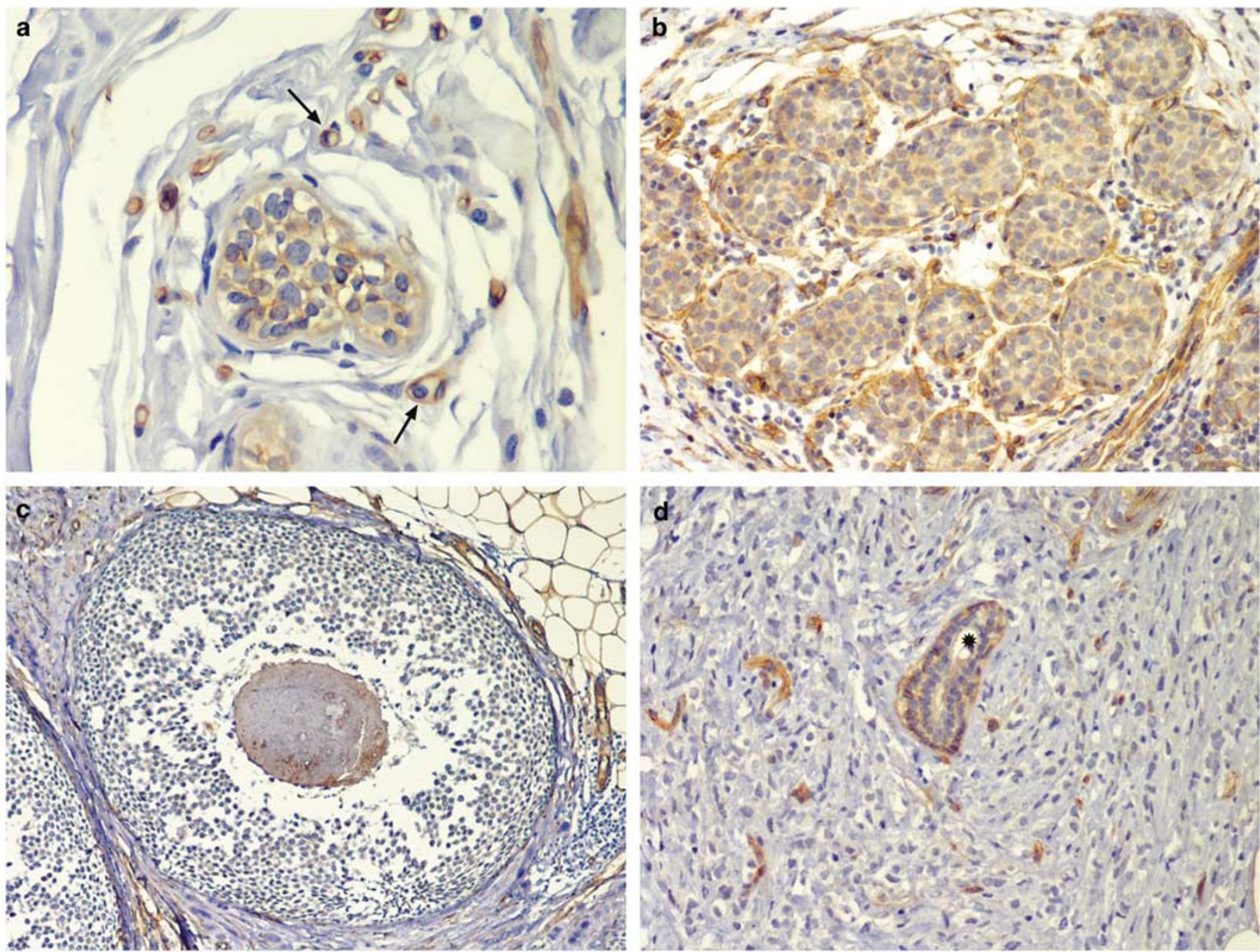

Figure 2 Cav-1 immunostaining. IHC was performed by the streptoavidin-biotin method. Cav-1 was expressed with a moderate/strong immunostaining in 92\% lobular intraepithelial neoplasia grade 1 (a), 68\% grade 2 (b) whereas $65 \%$ grade 3 (c) and $77 \%$ invasive lobular carcinoma (d) proved to be negative. In all cases examined, stromal tissue was negative, whereas normal ductal (asterisk) and lobular epithelial cells and blood vessel endothelial cells (arrows) were positive for Cav-1; thus, they were used as positive internal control. Magnification: (a) $\times 400$; (b, d) $\times 200 ;(\mathbf{c}) \times 100$.

Table 3 Cav-1 expression between lobular intraepithelial neoplasia with and without invasive lobular carcinoma

\begin{tabular}{|c|c|c|c|c|c|c|c|c|c|c|}
\hline & & \multicolumn{4}{|c|}{ Cav-1 in LIN without ILC } & \multicolumn{4}{|c|}{ Cav-1 in LIN with ILC } & \multirow[t]{2}{*}{$\mathrm{P}^{*}$} \\
\hline & & $\begin{array}{c}0 \\
\mathrm{n}(\%)\end{array}$ & $\begin{array}{c}1 \\
\mathrm{n}(\%)\end{array}$ & $\begin{array}{c}2 \\
\mathrm{n}(\%)\end{array}$ & $\begin{array}{c}3 \\
\mathrm{n}(\%)\end{array}$ & $\begin{array}{c}0 \\
\text { n }(\%)\end{array}$ & $\begin{array}{c}1 \\
\text { n }(\%)\end{array}$ & $\begin{array}{c}2 \\
\text { n }(\%)\end{array}$ & $\begin{array}{c}3 \\
\mathrm{n}(\%)\end{array}$ & \\
\hline Total LIN & 81 & $6(7)$ & 7 (9) & $11(14)$ & $22(27)$ & $9(11)$ & $5(6)$ & $4(5)$ & $17(21)$ & NS; 0.835 \\
\hline LIN grade 1 & 23 & $0(0)$ & $1(4)$ & $7(30)$ & $9(39)$ & $1(4)$ & $0(0)$ & $0(0)$ & $5(22)$ & NS; 0.157 \\
\hline LIN grade 2 & 38 & $2(5)$ & $4(11)$ & $4(11)$ & $12(32)$ & $4(11)$ & $2(5)$ & $1(3)$ & $9(24)$ & NS; 0.721 \\
\hline LIN grade 3 & 20 & $4(20)$ & $2(10)$ & $0(0)$ & $1(5)$ & $4(20)$ & 3 (15) & 3 (15) & 3 (15) & NS; 0.281 \\
\hline
\end{tabular}

LIN, lobular intraepithelial neoplasia; ILC, invasive lobular carcinoma; NS, not significant.

${ }^{*}$ Mann-Whitney test.

no significant differences emerged between lobular intraepithelial neoplasia grade 1 and lobular intraepithelial neoplasia grade 2 lesions $(P=0.306)$ whereas significant differences resulted between lobular intraepithelial neoplasia grade $3 \mathrm{vs}$ lobular intraepithelial neoplasia grade 1 and lobular intraepithelial neoplasia grade 2 (respectively, $P=0.0001$ and $P=0.007$; Table 4).

\section{Cav-1 in Invasive Lobular Carcinoma and Association with Lobular Intraepithelial Neoplasia}

No significant difference $(P=0.284)$, in terms of Cav-1 expression, emerged between invasive lesions with and without in situ component. Moreover, no statistically significant difference resulted by comparing Cav-1 expression with the 
Table 4 Cav-1 expression in lobular intraepithelial neoplasia lesions

\begin{tabular}{|c|c|c|c|c|c|c|}
\hline \multirow[t]{2}{*}{ Total LIN } & \multirow[b]{2}{*}{81} & \multicolumn{4}{|c|}{ Cav-1 expression } & \multirow[t]{2}{*}{$\mathrm{P}^{*}$} \\
\hline & & $\begin{array}{c}0 \\
\mathrm{n}(\%)\end{array}$ & $\begin{array}{c}1 \\
\mathrm{n}(\%)\end{array}$ & $\begin{array}{c}2 \\
\mathrm{n}(\%)\end{array}$ & $\begin{array}{c}3 \\
\mathrm{n}(\%)\end{array}$ & \\
\hline LIN grade 1 & 23 & $1(4)$ & $1(4)$ & $7(30)$ & $14(61)$ & LIN grade 1 vs grade $2:$ NS; 0.306 \\
\hline LIN grade 2 & 38 & $6(16)$ & $6(16)$ & $5(13)$ & $21(55)$ & LIN grade 1 vs grade 3: 0.0001 \\
\hline LIN grade 3 & 20 & $8(40)$ & $5(25)$ & $3(15)$ & $4(20)$ & LIN grade 2 vs grade 3: 0.007 \\
\hline
\end{tabular}

LIN, lobular intraepithelial neoplasia; NS, not significant.

*Mann-Whitney test.

Table 5 Cav-1 expression between invasive lobular carcinoma with and without lobular intraepithelial neoplasia association

\begin{tabular}{|c|c|c|c|c|c|c|}
\hline \multirow[t]{2}{*}{ Total ILC } & \multirow[b]{2}{*}{66} & \multicolumn{4}{|c|}{ Cav-1 expression } & \multirow[t]{2}{*}{$\mathrm{P}^{*}$} \\
\hline & & $\begin{array}{c}0 \\
\mathrm{n}(\%)\end{array}$ & $\begin{array}{c}1 \\
\mathrm{n}(\%)\end{array}$ & $\begin{array}{c}2 \\
\mathrm{n}(\%)\end{array}$ & $\begin{array}{c}3 \\
\text { n }(\%)\end{array}$ & \\
\hline ILC without LIN & 31 & $21(68)$ & $2(6)$ & $1(3)$ & $6(19)$ & NS; 0.2 \\
\hline ILC with LIN & 35 & $17(49)$ & $10(29)$ & $3(9)$ & $5(14)$ & \\
\hline LN neg. & 40 & $21(52)$ & $9(22)$ & $4(10)$ & $6(15)$ & NS; 0.42 \\
\hline LN pos. & 26 & $17(65)$ & 4 (15) & $0(0)$ & 5 (19) & \\
\hline
\end{tabular}

LIN, lobular intraepithelial neoplasia; ILC, invasive lobular carcinoma; LN, lymph nodes; NS, not significant; neg., negative; pos., positive.

*Mann-Whitney test.

presence of lymph node metastasis $(P=0.423$; Table 5).

Interestingly, significant statistical differences in terms of Cav-1 expression were established between lobular intraepithelial neoplasia lesions and invasive lobular carcinoma $(P=0.0001$; Figure 3$)$.

Moreover, evaluating Cav-1 expression in invasive lobular carcinoma lesions and comparing with the different grades of lobular intraepithelial neoplasia, significant statistical differences were found between lobular intraepithelial neoplasia grades 1 and 2 vs invasive lobular carcinoma (both, $P=0.0001$ ), whereas no difference was established between lobular intraepithelial neoplasia grade 3 and invasive lobular carcinoma $(P=0.196$; Table 6 , Figure 4$)$.

\section{Discussion}

In the current study, Cav-1 expression was evaluated in the whole spectrum of human lobular breast neoplasia and, interestingly, a progressive downexpression of Cav-1 was found between normal lobular epithelia and lobular intraepithelial neoplasia lesion and between lobular intraepithelial neoplasia lesion and invasive lobular carcinoma.

The term lobular breast neoplasia defines a continuum of diseases, from risk indicators for malignant disease through to lesions that are fully malignant and can lead to death for the patient. ${ }^{25}$ An

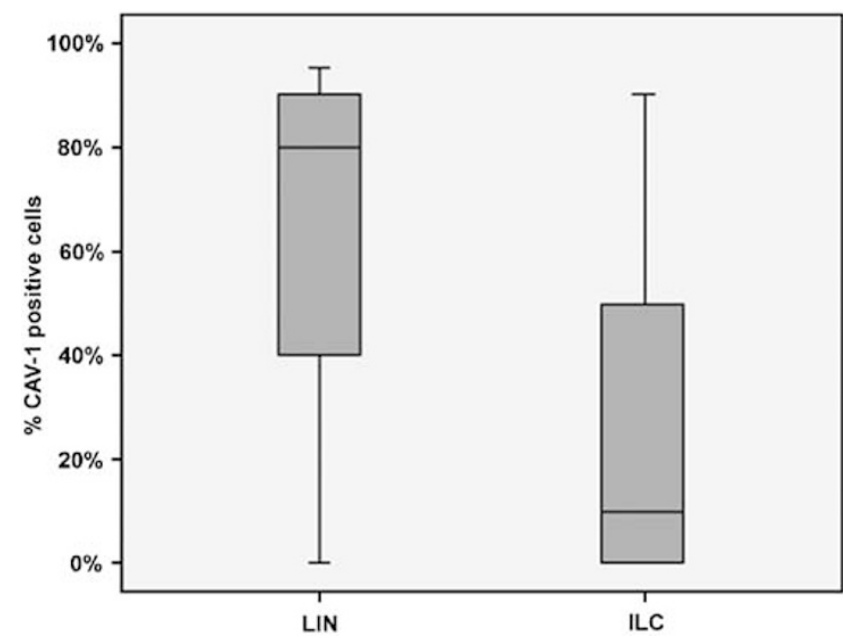

Figure 3 Cav-1 expression difference between lobular intraepithelial neoplasia and invasive lobular carcinoma. Significant statistical differences were found between lobular intraepithelial neoplasia lesions and invasive lobular carcinoma $(P=0.0001)$.

attempt to stratify the pre-malignant continuum on a logical morphological basis has been made with 'lobular intraepithelial neoplasia' terminology. Page et $a 1^{26}$ placed lobular intraepithelial neoplasia lesions into two categories: atypical lobular hyperplasia and lobular in situ carcinoma, on the basis of the percentage of acini distended and filled by lobular cells in a lobular unit. The risk factors for either one of the two are not sufficiently different, and the tendency toward invasive cancer development is not necessarily increased with the lobular in situ carcinoma designation. ${ }^{22}$ Other pathologists prefer to place lobular proliferative lesions in three different categories. Bratthauer and Tavassoli ${ }^{22}$ subdivided lobular intraepithelial neoplasia into three grades (lobular intraepithelial neoplasia grades 1-3) to reflect the spectrum of architectural and cytological changes that occur in this type of lesion. Using this classification system, Bratthauer and Tavassoli ${ }^{22}$ demonstrated that the increasing grade of lobular intraepithelial neoplasia directly correlates with the frequency of invasive lobular carcinoma.

Consistent with this study, our results showed a significant positive statistical correlation between 
Table 6 Cav-1 expression in lobular neoplastic lesions

\begin{tabular}{|c|c|c|c|c|c|c|}
\hline \multirow[t]{2}{*}{ Total LNs } & \multirow[t]{2}{*}{147} & \multicolumn{4}{|c|}{ Cav-1 expression } & \multirow[t]{2}{*}{$\mathrm{P}^{*}$} \\
\hline & & $\begin{array}{c}0 \\
n(\%)\end{array}$ & $\begin{array}{c}1 \\
n(\%)\end{array}$ & $\begin{array}{c}2 \\
n(\%)\end{array}$ & $\begin{array}{c}3 \\
n(\%)\end{array}$ & \\
\hline LIN grade 1 & 23 & $1(4)$ & $1(4)$ & $7(30)$ & $14(61)$ & LIN grade 1 vs ILC: 0.0001 \\
\hline LIN grade 2 & 38 & $6(16)$ & $6(16)$ & $5(13)$ & $21(55)$ & LIN grade 2 vs ILC: 0.0001 \\
\hline LIN grade 3 & 20 & $8(40)$ & $5(25)$ & $3(15)$ & $4(20)$ & LIN grade 3 vs ILC: NS; 0.196 \\
\hline ILC & 66 & $38(58)$ & $13(20)$ & $4(6)$ & $11(17)$ & \\
\hline
\end{tabular}

LNs, lobular neoplasias; LIN, lobular intraepithelial neoplasia; ILC, invasive lobular carcinoma; NS, not significant.

*Mann-Whitney test.

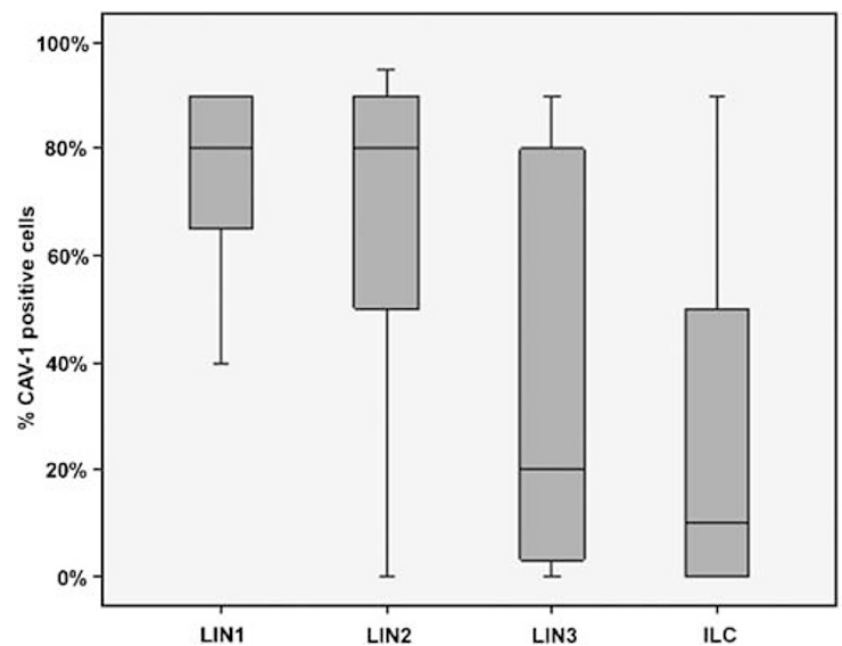

Figure 4 Cav-1 expression differences among lobular intraepithelial neoplasia grades and invasive lobular carcinoma. No differences emerged between lobular intraepithelial neoplasia grade 1 and lobular intraepithelial neoplasia grade 2 lesions $(P=0.306)$ and between lobular intraepithelial neoplasia grade 3 and invasive lobular carcinoma $(P=0.196)$ whereas a significant differences were found between lobular intraepithelial neoplasia grades 1 and 2 vs invasive lobular carcinoma (both, $P=0.0001$ ) and between lobular intraepithelial neoplasia grade 3 vs lobular intraepithelial neoplasia grades 1 and 2 (respectively, $P=0.0001$ and $P=0.007)$.

lobular intraepithelial neoplasia grade and invasive lobular carcinoma suggesting the potential clinical role of the 'lobular intraepithelial neoplasia' classification according to Tavassoli grade system for lobular intraepithelial neoplasia. This finding provides strong evidence for a precursor role of lobular intraepithelial neoplasia and supports the theory of the direct origin of invasive lobular carcinoma from clonal expansion of the cells found in lobular intraepithelial neoplasia lesions. ${ }^{27}$

Recently, many studies have showed that the D7S522 locus on human chromosome 7q31.1 is commonly deleted in a variety of human cancers, including carcinomas of the breast. These findings have led to the hypothesis that this region encodes a novel tumour suppressor gene. ${ }^{11-15,28,29}$ Moreover, the human Cav-1 gene was mapped to the D7S522 region of $7 \mathrm{q} 31.1{ }^{10}$ suggesting that $\mathrm{Cav}$-1 may indeed represent the tumour suppressor in this fragile genomic region.
Interestingly, Cav-1 is most highly expressed in terminally differentiated cells, such as adipocytes, endothelial cells, smooth muscle cells and mammary epithelial cells, ${ }^{2,3,5,30}$ suggesting that high levels of this protein may contribute to their well differentiated/non-proliferating state. ${ }^{9}$ These data were confirmed by our immunohistochemical analysis in which expression of Cav-1 was found in the normal mammary epithelia and surrounding endothelial cells of all breast tissue studied.

To date, only limited studies have investigated the Cav-1 expression in human breast cancer tissue; Park et $a l^{20}$ by immunohistochemical methods, demonstrated a reduction in Cav-1 expression in invasive ductal carcinoma of the breast compared with normal mammary epithelia. Furthermore, our group recently demonstrated the expression of Cav-1 protein in a large portion of human lobular intraepithelial neoplasia of the breast. ${ }^{21}$ Analysing Cav-1 expression in these three population, no significant differences were found between lobular intraepithelial neoplasia lesion with and without invasive component and between invasive lobular carcinoma with and without lobular intraepithelial neoplasia component. These data suggest that, at least for Cav-1, there is no association between Cav-1 expression in lobular intraepithelial neoplasia lesion and in the adjacent invasive lobular carcinoma.

Interestingly, a progressive downexpression of Cav-1 was found between normal lobular epithelia and lobular intraepithelial neoplasia lesion and between lobular intraepithelial neoplasia lesion and invasive lobular carcinoma. This data are consistent with the above in vitro data, which have shown that Cav-1 acts as a tumour suppressor in breast carcinogenesis. ${ }^{31,32}$

Moreover, in the current study, lobular intraepithelial neoplasia were stratified following Bratthauer and Tavassoli's classification, ${ }^{22}$ in a threetiered grading system and Cav-1 expression was evaluated among the three different grades of lobular intraepithelial neoplasia. No significant difference was found between lobular intraepithelial neoplasia grades 1 and 2 in terms of Cav-1 expression, whereas a significant difference was found between lobular intraepithelial neoplasia grades 1 and 3 and between lobular intraepithelial 
neoplasia grades 2 and 3 . Our results demonstrate that lobular intraepithelial neoplasia grade 3 lesions show different Cav-1 expression from lobular intraepithelial neoplasia grades 1 and 2 lesions, allowing the hypothesis that this different pattern of protein expression reflects a different biological behaviour. Furthermore, a significant difference was found between lobular intraepithelial neoplasia grades 1-2 and invasive lobular carcinoma whereas no difference was established between lobular intraepithelial neoplasia grade 3 and invasive lobular carcinoma. In a recent report, evaluating expression of Cox-2, an enzymatic protein linked to the development of breast cancer, similar results were presented. ${ }^{33}$ Take together, a provocative possible explanation is that lobular intraepithelial neoplasia grade 3 , rather than grades 1 and 2 lesions, is a precursor lesion of invasive lobular carcinoma rather than a simple risk factor.

In summary, data presented here confirm and extend the in vitro findings of others and show for the first time that Cav-1 may have a key role as a tumour suppressor in the lobular breast carcinogenesis. In future, it will be important to extend these observations in further investigation to better understand the pathways and processes that are involved in the development and progression of lobular breast cancer, thus to offer exciting opportunities to develop new anti-cancer therapies.

\section{Disclosure/conflict of interest}

The authors declare no conflict of interest.

\section{References}

1 Okamoto T, Schlegel A, Scherer PE, et al. Caveolins, a family of scaffolding proteins for organizing 'preassembled signaling complexes' at the plasma membrane. J Biol Chem 1998;273:5419-5422.

2 Lisanti MP, Scherer PE, Tang Z, et al. Caveolae, caveolin and caveolin-rich membrane domains: a signalling hypothesis. Trends Cell Biol 1994;4:231-235.

3 Lee SW, Reimer CL, Oh P, et al. Tumor cell growth inhibition by caveolin re-expression in human breast cancer cells. Oncogene 1998;16:1391-1397.

4 Sager R, Sheng S, Anisowicz A, et al. RNA genetics of breast cancer: maspin as paradigm. Cold Spring Harb Symp Quant Biol 1994;59:537-546.

5 Engelman JA, Lee RJ, Karnezis A, et al. Reciprocal regulation of neu tyrosine kinase activity and caveolin-1 protein expression in vitro and in vivo. Implications for human breast cancer. J Biol Chem 1998;273: 20448-20455.

6 Engelman JA, Zhang XL, Lisanti MP. Sequence and detailed organization of the human caveolin-1 and -2 genes located near the D7S522 locus (7q31.1). Methylation of a $\mathrm{CpG}$ island in the $5^{\prime}$ promoter region of the caveolin-1 gene in human breast cancer cell lines. FEBS Lett 1999;448:221-230.
7 Cohen AW, Hnasko R, Schubert W, et al. Role of caveolae and caveolins in health and disease. Physiol Rev 2004;84:1341-1379.

8 Engelman JA, Zhang XL, Galbiati F, et al. Chromosomal localization, genomic organization, and developmental expression of the murine caveolin gene family (Cav-1, -2, and -3). Cav-1 and and Cav-2 genes map to a known tumor suppressor locus (6-A2/7q31). FEBS Lett 1998;429:330-336.

9 Engelman JA, Zhang X, Galbiati F, et al. Molecular genetics of the caveolin gene family: implications for human cancers, diabetes, Alzheimer disease, and muscular dystrophy. Am J Hum Genet 1998;63:1578-1587.

10 Engelman JA, Zhang XL, Lisanti MP. Genes encoding human caveolin-1 and -2 are co-localized to the D7S522 locus (7q31.1), a known fragile site (FRA7G) that is frequently deleted in human cancers. FEBS Lett 1998;436:403-410.

11 Zenklusen JC, Bièche I, Lidereau R, et al. (C-A)n microsatellite repeat D7S522 is the most commonly deleted region in human primary breast cancer. Proc Natl Acad Sci USA 1994;91:12155-12158.

12 Zenklusen JC, Thompson JC, Troncoso P, et al. Loss of heterozygosity in human primary prostate carcinomas: a possible tumor suppressor gene at 7q31.1. Cancer Res 1994;54:6370-6373.

13 Zenklusen JC, Thompson JC, Klein-Szanto AJ, et al. Frequent loss of heterozygosity in human primary squamous cell and colon carcinomas at $7 \mathrm{q} 31.1$ : evidence for a broad range tumor suppressor gene. Cancer Res 1995;55:1347-1350.

14 Kerr J, Leary JA, Hurst T, et al. Allelic loss on chromosome $7 \mathrm{q}$ in ovarian adenocarcinomas: two critical regions and a rearrangement of the PLANH1 locus. Oncogene 1996;13:1815-1818.

15 Shridhar V, Sun QC, Miller OJ, et al. Loss of heterozygosity on the long arm of human chromosome 7 in sporadic renal cell carcinomas. Oncogene 1997;15:2727-2733.

16 Jenkins RB, Qian J, Lee HK, et al. A molecular cytogenetic analysis of 7q31 in prostate cancer. Cancer Res 1998;58:759-766.

17 Lee H, Park DS, Razani B, et al Caveolin-1 mutations (P132L and null) and the pathogenesis of breast cancer: caveolin-1 (P132L) behaves in a dominant-negative manner and caveolin-1 (-/-) null mice show mammary epithelial cell hyperplasia. Am J Pathol 2002;161:1357-1369.

18 Park DS, Lee H, Riedel C, et al. Prolactin negatively regulates caveolin-1 gene expression in the mammary gland during lactation, via a Ras-dependent mechanism. J Biol Chem 2001;276:48389-48397.

19 Park DS, Lee H, Frank PG, et al. Caveolin-1-deficient mice show accelerated mammary gland development during pregnancy, premature lactation, and hyperactivation of the Jak-2/STAT5a signaling cascade. Mol Biol Cell 2002;13:3416-3430.

20 Park SS, Kim JE, Kim YA, et al. Caveolin-1 is downregulated and inversely correlated with HER2 and EGFR expression status in invasive ductal carcinoma of the breast. Histopathology 2005;47:625-630.

21 Perrone G, Zagami M, Altomare V, et al. COX-2 localization within plasma membrane caveolae-like structures in human lobular intraepithelial neoplasia of the breast. Virchows Arch 2007;451:1039-1045.

22 Bratthauer GL, Tavassoli FA. Lobular intraepithelial neoplasia: previously unexplored aspects assessed in 
775 cases and their clinical implications. Virchows Arch 2002;440:134-138.

23 Sotgia F, Williams TM, Schubert W, et al. Caveolin-1 deficiency $(-/-)$ conveys premalignant alterations in mammary epithelia, with abnormal lumen formation, growth factor independence, and cell invasiveness. Am J Pathol 2006;168:292-309.

24 Salatino M, Beguelin W, Peters MG, et al. Progestininduced caveolin-1 expression mediates breast cancer cell proliferation. Oncogene 2006;25:7723-7739.

25 Hanby AM, Hughes TA. In situ and invasive lobular neoplasia of the breast. Histopathology 2008;52:58-66.

26 Page DL, Dupont WD, Rogers LW, et al. Atypical hyperplastic lesions of the female breast. A long-term follow-up study. Cancer 1985;55:2698-2708.

27 Vos CB, Cleton-Jansen AM, Berx G, et al. E-cadherin inactivation in lobular carcinoma in situ of the breast: an early event in tumorigenesis. $\mathrm{Br} \mathrm{J}$ Cancer 1997;76:1131-1133.

28 Matsuura K, Shiga K, Yokoyama J, et al. Loss of heterozygosity of chromosome 9p21 and 7q31 is correlated with high incidence of recurrent tumor in head and neck squamous cell carcinoma. Anticancer Res 1998;18:453-458.

29 Zenklusen JC, Weitzel JN, Ball HG, et al. Allelic loss at 7q31.1 in human primary ovarian carcinomas suggests the existence of a tumor suppressor gene. Oncogene 1995;11:359-363.

30 Scherer PE, Tang Z, Chun M, et al. Caveolin isoforms differ in their N-terminal protein sequence and subcellular distribution. Identification and epitope mapping of an isoform-specific monoclonal antibody probe. J Biol Chem 1995;270:16395-16401.

31 Razani B, Schlegel A, Liu J, et al. Caveolin-1, a putative tumour suppressor gene. Biochem Soc Trans 2001;29:494-499.

32 Sagara Y, Mimori K, Yoshinaga K, et al. Clinical significance of Caveolin-1, Caveolin-2 and HER2/neu mRNA expression in human breast cancer. Br J Cancer 2004;91:959-965.

33 Perrone G, Zagami M, Santini D, et al. COX-2 expression in lobular in situ neoplasia of the breast: correlation with histopathological grading system according to the Tavassoli classification. Histopathology 2007;51:33-39. 